\title{
O que o profissional da saúde precisa saber a respeito do atendimento às pessoas transexuais ou transgênero
}

Kadija Rahal Chrisostomo ${ }^{1}$ (D), Bernardo Passos Sobreiro ${ }^{3}$ (D), Eduardo Rahal Chrisostomo ${ }^{2}$ (D), Renato Mitsunori Nisihara ${ }^{1,2,3}$ [D

\section{RESUMO}

Introdução: As pessoas transexuais encontram uma série de dificuldades no atendimento médico, tanto na rede privada como na pública no Brasil.

Objetivo: Demonstrar aos profissionais da área da saúde a realidade vivida pelas pessoas transexuais, quando necessitam de atendimento médico no Brasil, visando aprimorar os conhecimentos sobre o atendimento a este grupo populacional.

Métodos: Coleta de dados realizada em fevereiro de 2020 com buscas nas bases eletrônicas: PubMed, Scielo, Lilacs e Google Scholar. Utilizou-se os descritores em associação: transgender; medical care; transexual; cross-sex hormone therapy, no período entre 2000 a 2020.

Resultados: Encontramos 720 publicações com as palavras-chave transgender persons e medical care, 30 com as palavras-chave transgender, medical care e cross-sex hormone therapy, sendo escassos os estudos feitos no Brasil. Conclusão: Para que ocorra uma melhora no atendimento à saúde das pessoas transexuais é necessário que sejam inseridas disciplinas específicas sobre este tema na grade curricular dos cursos técnicos, de graduação e de pós-graduação da área da saúde.

Palavras-chave: Pessoas transgênero, Pessoas transexual, Cuidados médicos, Terapia de reposição hormonal.

1. Universidade Federal do Paraná. Programa de Pós-graduação em Medicina Interna e Ciências da Saúde, Curitiba, (PR), Brasil

2. Faculdade Evangélica Mackenzie. Departamento de Medicina, Curitiba, (PR), Brasil

3. Universidade Positivo. Departamento de Medicina, Curitiba, (PR), Brasil 


\section{INTRODUÇÃO}

Historicamente, a transexualidade sempre existiu, porém, no século $X X$, com advento da terapia hormonal ( $\mathrm{TH}$ ) e procedimentos cirúrgicos específicos, as pessoas trans passaram a adquirir forma física muito semelhante à mulher ou homem cisgênero ${ }^{1-3}$. O que determina a Identidade de Gênero para a pessoa transexual é a forma como ela se identifica, independente se realizou ou não os procedimentos cirúrgicos como: mastectomia, histerectomia, ooforectomia, tireoplastia, cirurgia de afirmação de sexo, procedimentos estéticos e cirurgias plásticas.

O conceito de gênero e identidade de gênero vem sendo amplamente discutido, importantes autores como Joan Scott, que em sua obra afirma que codificar o sentido das palavras é praticamente impossível, pois como as ideias e as coisas que elas pretendem significar tem uma história e dependem da época, cultura e situação em que são empregadas ${ }^{4}$.

Já Judith Butler, aborda o conceito que hoje é a definição do espectro de gênero, criticando a construção da identidade como algo finito e de caráter determinista. Entende-se que esse processo seja contínuo, manifestado através da sua expressão de gênero. A autora coloca em xeque o binarismo latente, atestando assim sua fragilidade frente a um mundo que sempre foi diverso e plural ${ }^{5}$.

A transexualidade é uma experiência que está localizada no gênero; a cirurgia de afirmação de sexo e/ou terapias hormonais não definem a orientação sexual da pessoa, segundo Berenice Bento ${ }^{6}$.

O número de pessoas transexuais que procuram atendimento médico parece estar aumentando em todo mundo ${ }^{3}$. Ainda é incerto quantos indivíduos experimentam a transexualidade durante a vida. Estima-se que seja em torno de 4,6 em 100.000 pessoas, porém, provavelmente este número esteja subestimado7. Em estudo recente, sugere que a população de transexuais nos EUA esteja em torno de 1 milhão de adultos ${ }^{8}$. No Brasil não há dados que permitam quantificar esta população com precisão.

A identidade de gênero (IG) é como a pessoa se vê ou se percebe. Há uma ampla gama de conceitos e termos relacionados a IG, que pode ocasionar interpretações errôneas. No Brasil, não há um consenso sobre os termos. Os mais aceitos e utilizados são: masculino e feminino para definir o sexo biológico, relacionados aos cromossomos, gônadas e conformação genital. Porém, a IG é construída socialmente, pelas diferenças culturais, determinando o que é ser homem ou mulher. Assim, todos podem ser enquadrados como cisgêneros, quando a IG é a mesma do sexo biológico ou transgêneros, quando há uma incongruência a respeito do sexo de nascimento ${ }^{9}$. (Quadro 1.)

A orientação sexual se refere à atração afetivo sexual e por alguém de algum(ns) gênero(s). Não há uma norma de orientação sexual em função do gênero das pessoas ${ }^{10}$.

Atualmente, considera-se mulher transexual: toda pessoa que embora tenha nascido como do sexo biológico masculino reivindica o reconhecimento social e legal como mulher, ou seja, como gênero feminino. Considera-se homem transexual: toda pessoa que tenha nascido como do sexo biológico feminino reivindica o reconhecimento social e legal como homem, ou seja, gênero masculino ${ }^{11}$.

Desde 1980, a transexualidade era considerada um transtorno disfórico pela Organização Mundial da Saúde, presente na Classificação Estatística Internacional de Doenças e Problemas Relacionados a Saúde (CID 10), sob o número F64.0, no capítulo $\mathrm{V}$ que abrangia transtornos mentais e comportamentais, gerando muita discussão, revolta e indignação da comunidade transexual e pessoas cientes do que é a transexualidade ${ }^{12}$. Em 18 de junho de 2018, a Organização Mundial da Saúde (OMS) lançou a nova classificação, o CID 11, que entrará em vigor em $1^{\circ}$ de janeiro de 2022. Nesta nova classificação, a transexualidade sai da lista de doenças mentais, em que era classificada como disforia de gênero, e entra em uma nova categoria denominada condições relacionadas à saúde sexual, mais especificamente descrita como incongruência de gênero sob os códigos: HA60 (Incongruência de gênero do adolescente ou do adulto), HA61 (Incongruência de gênero da infância) e HA6Z (Incongruência de gênero, inespecífica) ${ }^{13}$.

No dia 09/01/2020, o Conselho Federal de Medicina do Brasil atualizou parâmetros para atendimento da população transexual do país ${ }^{14}$. A resolução CFM n.o 2.265/2019 prevê a ampliação do acesso ao atendimento desta população, estabelecendo critérios para uma maior segurança na realização de tratamentos com hormônios e procedimentos cirúrgicos para a adequação sexual. Os protocolos adotados estão mais próximos aos da Endocrine Society ${ }^{15}$. 
Quadro 1. Principais termos empregados para identidade de gênero

\section{TERMOS RELACIONADOS}

Sexo

Gênero

Expressão de Gênero

Transgênero

Transexualidade

Transexual

Mulher Trans

Homem Trans

Gay

Lesbianidade

Homossexualidade

Crossdressing

Drag queen

Drag king

Travesti

Não-binário

Afirmação de gênero

Orientação sexual

Queer

Intersexual

Assexual

LGBTQIA+

\section{DESCRIÇÃO}

\section{Fenótipo biológico presente ao nascimento}

Feminino / Masculino

Como a pessoa se vê ou se percebe

Mulher / Homem / Neutro / Bigênero

Como a pessoa se expressa, se veste ou se apresenta através do tipicamente masculino, feminino ou neutro

Incongruência pessoal com o próprio sexo biológico (presente ao nascimento)

É transição social pela qual a pessoa transgênero pode passar, através da expressão de gênero ou ainda, se desejar, tratamentos hormonais e/ou cirúrgicos

É a pessoa transgênero que passa pelo processo de transexualidade

Biologicamente homem que se percebe como mulher

Biologicamente mulher que se percebe como homem

Atração erótica e afetiva por pessoa do mesmo sexo

Atração erótica e afetiva de uma mulher por outra

o homem ou a mulher cujo desejo erótico e afetivo é voltado a indivíduos do mesmo sexo

Veste-se com roupas do sexo oposto em ocasiões específicas

Homem que se veste como mulher de uma forma caricata para realizar performances artísticas (canto e dança)

Mulher que se veste como homem de uma forma caricata para realizar performances artísticas (canto e dança)

Conceito que apresenta divergências, é um homem que usa roupas e/ou hormônios femininos e não sente desconforto com a sua genitália

Quando a identidade de gênero não é masculina nem feminina, uma combinação dos dois ou ausência (neutralidade)

Procedimento multidisciplinar que possibilita a pessoa trans adequar seu corpo a sua identidade de gênero

É independente do sexo ou gênero, é a atração afetiva

Indivíduo que não corresponde a um padrão heteronormativo (sexo biológico, orientação sexual, identidade de gênero e expressão de gênero)

Indivíduo que está na variável entre os dois espectros de gênero, qualquer variação dos caracteres sexuais, dificultando a identificação do indivíduo como totalmente masculino ou feminino

Indivíduo que não tem interesse sexual, termo ainda não muito discutido

Lésbicas, gays, bissexuais, transexuais (ou travestis), queer, intersexo, assexuais e outros

Elaborado pelos autores

Ocorreram três grandes mudanças nesta resolução:

1. Inclusão de todos os procedimentos e respectivos protocolos de acompanhamento (psicológico, hormonal e cirúrgico);

2. Normatização do tratamento em crianças e adolescentes, em que o conselho aprova o acompanhamento;

3. Processo terapêutico singular (individualizado).
4. As pessoas transexuais, devido à incongruência com o seu gênero e/ou sexo de nascimento, enfrentam um preconceito social estigmatizado e, geralmente, há falta de apoio familiar, ocasionando inúmeros prejuízos a sua saúde e condição social. Inicialmente com dificuldade de aprendizagem e elevados índices de evasão escolar, prejudicando o seu futuro e sua condição socioeconômica. As pessoas 
transexuais podem sentir desconforto ou angústia quando as oportunidades para expressar essa identidade são negadas ou onde essa identidade não é respeitada. Muitas vezes, ficam à margem da sociedade, em situações de risco. Em grande parte do mundo, pessoas transexuais têm dificuldade em acessar e/ou utilizar cuidados de saúde de boa qualidade, sejam eles específicos para necessidades de gênero ou de natureza mais geral ${ }^{12}$.

A assistência à saúde desta população deve ser realizada por uma equipe multidisciplinar composta por: médicos, psicólogos, enfermagem e atendentes capacitados a receber, acolher e atendê-las(Ios). Quando há o correto atendimento e apoio social, os sintomas são significativamente atenuados, apresentando melhor qualidade de saúde mental e maior aderência ao atendimento, de modo psicossocial ${ }^{16,17}$. No entanto, de maneira geral, pessoas transexuais enfrentam alguns desafios ao procurar serviços de saúde, em comparação com as pessoas cisgêneros, pois a maioria dos profissionais de saúde não tem formação adequada prévia, devido ao fato deste conteúdo não fazer parte da grade curricular dos cursos da área da saúde, resultando em inaptidão por parte dos profissionais diante da necessidade de prestar esse atendimento.

Este artigo tem como objetivo demonstrar aos profissionais da área da saúde a realidade vivida pelas pessoas transexuais quando necessitam de atendimento médico no Brasil, visando aprimorar os conhecimentos sobre $o$ atendimento a este grupo populacional.

\section{MATERIAL E MÉTODOS}

Foi realizada uma pesquisa nas bases eletrônicas de dados: PubMed - MEDLINE (Medical Literature Analysis and Retrievel System Online), SciELO (Scientific Electronic Library Online) e LILACS (Literatura Latino-Americana e do Caribe em Ciências da Saúde) em fevereiro de 2020. Utilizou-se os descritores associados: transgender; medical care; transsexual; cross-sex hormone therapy e Brasil, no período entre 2000 e 2020, encontramos 720 publicações. Quando utilizamos bases de dados não indexadas, como o Google Scholar, encontramos mais de 69 mil publicações.

\section{RESULTADOS}

\section{Atendimento médico no Sistema Único de Saúde (SUS)}

No Brasil, existem nove centros de atendimento pelo SUS que realizam o processo transexualizador (quatro centros localizados em Curitiba, Uberlândia, São Paulo e Rio de Janeiro, realizam somente tratamento ambulatorial com terapia hormonal e cinco centros localizados em São Paulo, Rio de Janeiro, Goiânia, Recife e Porto Alegre, realizam tratamento ambulatorial com terapia hormonal e tratamento cirúrgico, a cirurgia de afirmação de sexo), desde 19/11/2013, sob a portaria $2.803 / \mathrm{GM}^{18}$. Somente a região norte do país não conta com esse serviço. Todos os centros possuem equipe multidisciplinar composta por: médicos, enfermeiros e psicólogos. Há um protocolo de atendimento a ser seguido. A terapia hormonal (TH) fornecida pelo SUS para a mulher transexual é o estrogênio (Estradiol) e bloqueadores androgênicos (Acetato de Ciproterona ou Espironolactona). Para o homem transexual, a TH padrão é feita com Testosterona (Cipionato de Testosterona ou Undecanoato de Testosterona), ambos injetáveis ou Testosterona Gel para uso transdérmico.

Esses centros de atendimento oferecem assistência especializada às pessoas com indicação de realizar o processo transexualizador. Busca-se promover o bem-estar psicossocial e clínico dos/as pacientes durante seu processo de vivência e/ou adequação a sua identidade/expressão de gênero. Devem oferecer atendimento humanizado e multidisciplinar, podendo ou não culminar no processo de redesignação sexual. Diante da carência de estudos científicos e de profissionais capacitados para as especificidades de saúde e atendimento desta população no Brasil, estes centros desenvolvem ações de formação continuada de profissionais que ali trabalham ${ }^{19}$.

O Brasil é um dos países pioneiros no atendimento médico público direcionado às pessoas transexuais nos centros especializados. No entanto, fora desses locais, seja no atendimento ambulatorial geral e/ou de emergência, os profissionais não estão capacitados para tal atendimento e não existem treinamentos específicos. Ainda não faz parte da grade curricular dos cursos de graduação das áreas da saúde e nem das residências médicas. Nos últimos anos, diversos artigos foram publicados com o intuito de melhorar o atendimento à população transexual 20-22. 


\section{Atendimento médico privado}

A medicina privada no Brasil ainda não está preparada a atender o/a paciente transexual, seja por falta de conhecimento científico e de experiência. Na última década, algumas sociedades médicas elaboraram guias de conduta específicas ao tratamento de pessoas transexuais ${ }^{23}$ (Endocrine Society Clinical Practice Guideline, 2017) e diretrizes internacionais da Associação Profissional Mundial da Saúde Transgênero ${ }^{3}$ (The World Professional Association for Transgender Health, WPATH, 2012).

A/O paciente transexual necessita de um atendimento médico que avalie o estado geral de sua saúde e encaminhe para as especialidades quando for necessário. As orientações de conduta profissional devem abranger todas as pessoas que trabalham na área da saúde. Todos os colaboradores, desde a portaria, setores da segurança, limpeza, secretaria, enfermagem e outros. Todos devem estar preparados ao atendimento, respeitando, acolhendo de uma maneira cordial e natural. Ou seja, não deve haver discriminações, olhares indiscretos ou de curiosidade, sussurros ou piadinhas, perguntas impróprias e invasivas e evitar constrangimento público do(a) paciente. Há de ser respeitada a identidade/ expressão de gênero da pessoa transexual, de acordo com sua estética, seu desejo e sua reivindicação de como quer ser tratada.

Recomenda-se que após a realização do cadastro para consulta, na hora de chama-la(o) para o atendimento médico, a/o paciente deve ser chamada(o) pelo nome social (independente do nome que esteja na carteira de identidade, caso ainda não tenha feito a mudança dos documentos com seu atual status sexual e de gênero) $)^{24}$ e com naturalidade, evitando constrangimento. $\mathrm{O}$ médico assistente deve manter a mesma postura, sem demonstrar surpresa, curiosidade, preconceitos ou julgamentos. Para todo atendimento médico, independente das especialidades, a consulta deve ser exatamente igual a de um paciente cisgênero, com exceção dos especialistas envolvidos na afirmação de gênero, estes sim, precisam de todas as informações necessárias para prestar atendimento mais adequado possível (ginecologista, urologista e endocrinologista).

Caso na localidade do(a) paciente não tenha esses especialistas, o médico clínico geral é o mais indicado a realizar o tratamento ambulatorial. Quando o médico não se sentir preparado para o atendimento $\mathrm{da} / \mathrm{o}$ paciente transexual, ele pode comunicar a/o paciente que não tem experiência, que fará uma primeira consulta e no retorno ele estará mais preparado para finalizar este primeiro atendimento. $O$ profissional pode buscar informações, usando fontes seguras como: guideline da Associação Mundial Profissional para Saúde Transgênero - WPATH - World Professional Association for Transgender Health, 2012 ${ }^{3}$, Endocrine Society $2017^{23}$ e protocolos de atendimento a transexuais do Sistema Único de Saúde ${ }^{19}$, assim como na literatura ampliada de estudos e pesquisas com outras(os) profissionais que estudam o tema em áreas correlatas.

Resumindo, se a queixa da/do paciente não estiver relacionada a transexualidade, ela/ele deve ser tratada(o) como paciente cisgênero, por exemplo: foi ao ortopedista por uma entorse no tornozelo, a consulta fica somente relacionado a queixa, evitando questionamentos constrangedores e desnecessários para a situação apresentada.

Quando a consulta é marcada com profissionais com o objetivo de afirmação de sexo, a primeira consulta pode ser realizada de uma forma mais generalista, onde primeiro há a queixa, os questionamentos, as dúvidas da/do paciente, conforme esta sistemática segue a consulta com o objetivo de esclarecer, informar e orientar, por exemplo:

1. Queixa principal (especificações a respeito e perguntas habituais como: qual idade ele (a) percebeu a incongruência de gênero, se já fez ou faz algum tratamento, quais seus desejos, o que espera do tratamento, como prefere ser chamada(o), etc...);

2. Antecedentes mórbidos pessoais: o habitual, incluir história sexual, possíveis distúrbios psiquiátricos e experiências transfóbicas;

3. Antecedentes mórbidos familiares.

Ao exame físico, realizar o exame geral (peso, pressão arterial, frequência cardíaca, ausculta cardíaca, ausculta pulmonar, avaliação de mucosas e orofaringe, palpação de tireoide, linfonodos e abdômen). O exame mais específico em mamas e genitália, somente se a queixa principal está relacionada. Se necessário, pode-se deixar esta avaliação para a segunda consulta, para a/o profissional se preparar para um atendimento mais adequado. Sempre que possível, a/o médica/o 
deve estar acompanhada/o de algum profissional assistente ao realizar o exame físico.

Solicitar os exames complementares necessários na primeira consulta e segunda consulta, se necessário, solicitar exame mais específicos ou encaminhar para especialista (Quadro 2).

Quadro 2. Atendimento médico a/ao paciente transexual - Orientações por especialidades

\begin{tabular}{|c|c|}
\hline ESPECIALIDADE & DESCRIÇÃO \\
\hline Generalista & $\begin{array}{c}\text { Anamnese } \\
\text { Exame físico geral } \\
\text { Exame físico específico (somente } \\
\text { se relacionado à queixa principal) } \\
\text { e se não houver como encaminhar } \\
\text { ao especialista }\end{array}$ \\
\hline Ginecologista & $\begin{array}{c}\text { Anamnese } \\
\text { Exame físico geral } \\
\text { Exame físico específico } \\
\text { Mulher Trans: Mamas, neovagina e } \\
\text { tratamento hormonal } \\
\text { Homem Trans: Mamas, vulva, } \\
\text { vagina, útero e ovários }\end{array}$ \\
\hline Urologista & $\begin{array}{c}\text { Anamnese } \\
\text { Exame físico geral } \\
\text { Exame físico específico } \\
\text { Mulher Trans: órgãos genitais } \\
\text { externos e próstata } \\
\text { Homem Trans: Neofalo e } \\
\text { tratamento hormonal }\end{array}$ \\
\hline
\end{tabular}

Elaborado pelos autores

Caso a/o paciente transexual não possa ser atendida/o nos centros especializados ao atendimento a transexuais ou por médicos especializados da rede privada, o tratamento de afirmação de sexo pode ser realizado por médico clínico que deve seguir as orientações dos guidelines acima citados.

\section{Avaliação ginecológica}

Um estudo realizado em parceria com a American College of Obstetricians and Gynecologists, demonstrou que, no grupo de ginecologistas estudado, 57,4\% jamais tiveram treinamento para lidar com pessoas transexuais. Dos ginecologistas que já prestaram atendimento a pessoas trans, somente $18 \%$ tiveram disciplinas específicas durante a graduação ${ }^{25}$.

Acredita-se que a forma correta de aprimorar o atendimento às pessoas transexuais é a inclusão de disciplinas específicas nos currículos de graduação e programas de educação continuada, para toda a equipe multidisciplinar necessária ${ }^{22}$.

As mulheres transexuais necessitam de avaliação ginecológica para prevenção, diagnóstico e tratamento de enfermidades mamárias e em casos de pacientes submetidas à cirurgia de redesignação sexual, avaliar vagina e vulva.

E os homens transexuais que não realizaram cirurgias para exérese da glândula mamária, histerectomia, ooforectomia e cirurgia de redesignação sexual, também necessitam de acompanhamento ginecológico, considerando que o câncer de mama é a primeira causa mais frequente de morte no Brasil e o câncer de colo do útero é a quarta ${ }^{26}$.

\section{Avaliação urológica}

Os homens transexuais que realizaram a cirurgia de transexualização devem realizar acompanhamento clínico com o mesmo objetivo, prevenção, diagnóstico e tratamento de possíveis enfermidades.

As mulheres transexuais que não querem realizar ou que ainda não realizaram a cirurgia de transexualização, necessitam de consultas de rotina para prevenção, principalmente de câncer de próstata, dada sua alta prevalência ${ }^{26}$. Até o momento foram descritos 11 casos de câncer de próstata em mulheres transexuais ${ }^{27}$ Possivelmente tal número pode ser subestimado, tendo em vista a dificuldade de acesso destas mulheres ao atendimento especializado e outro fator seria pela vida média do paciente transexual ser menor e o câncer de próstata ser mais comum em indivíduos com idade mais avançada.

Uma questão a ser melhor compreendida é o papel do bloqueio hormonal de longa data na incidência do câncer de próstata nas mulheres trans. Do ponto de vista prático, o tratamento hormonal da mulher trans é similar ao bloqueio androgênico que se utiliza no câncer de próstata metastático. Assim, um tumor em fase inicial seria mantido em estado latente na mulher trans devido ao baixo nível de testosterona. E, como na doença metastática, em determinado momento esse tumor passará a ser resistente à cirurgia de afirmação de sexo e progredirá de forma mais agressiva se tornando clinicamente detectável. Esse dado pode ser observado no estudo de Ingham et al. ${ }^{28}$, onde das nove pacientes com estadiamento conhecido, seis $(66,7 \%)$ tinham doença metastática ao diagnóstico. 
De acordo com o National Cancer Institute (EUA), somente $4 \%$ dos homens da população norteamericana apresentam metástase no momento do diagnóstico do câncer de próstata ${ }^{29}$. Diante desse quadro, recomenda-se muita atenção à paciente transexual no rastreamento para câncer de próstata, principalmente se houver sintomas como dificuldade miccional, hematúria ou dor óssea.

Com o bloqueio androgênico de longa data são esperados nas mulheres trans sinais e sintomas típicos da deficiência de testosterona como diminuição da libido, aumento da gordura corporal, redução da massa muscular e óssea, alteração do humor, fadiga e anemia ${ }^{28}$. Esse é um cenário desafiador uma vez que a suspensão do bloqueio hormonal ou a reposição de testosterona não são indicadas para esse grupo de pacientes.

\section{Saúde Mental}

É muito comum as pessoas transexuais apresentarem alterações psiquiátricas de menor a maior grau de comprometimento, tais como: ansiedade, depressão, alteração de humor, bipolaridade e outras. Possivelmente devido a toda trajetória de vida que na maioria das vezes elas precisam enfrentar. Inicia-se quando começam a sentir a incongruência de gênero, que vem acompanhado de sentimentos confusos sobre seu corpo, sua sexualidade, seu comportamento e muitos questionamentos. A maioria vivencia tais experiências completamente sozinha, sem apoio familiar e sem conhecimento do que está acontecendo. Concomitantemente, já pode estar ocorrendo transfobia na escola, entre amigos e a não aceitação dos familiares.

Cada família e sociedade lida com a transexualidade de acordo com suas crenças e cultura. Na maioria dos casos, o que é oferecido à criança ou ao adolescente nesta fase de vida tão delicada é insuficiente. Elas/eles precisam de afeto, apoio, empatia e segurança para que sua afirmação de sexo e de gênero seja menos traumática possível. Dessa forma, se poderia tentar evitar o surgimento de muitos problemas psicológicos e psiquiátricos que irão refletir negativamente no seu futuro, tais como evasão escolar, dificuldade de aprendizado, de acesso ao trabalho, baixa autoestima, drogadição, ideação suicida, ocasionando uma baixa condição socioeconômica, ficando à margem da sociedade. Apenas para reforçar tal situação, no Brasil a expectativa de vida média dos transexuais é de apenas 35 anos, sendo associada à violência e ao suicídio ${ }^{29}$.

Nosso estudo reflete a realidade da maioria das/dos transexuais no Brasil. No entanto, há exceções onde a pessoa transexual teve aceitação, início de tratamento apropriado, acompanhamento em todas as esferas, inclusive acesso à formação escolar e laboral, consequentemente preservando seu futuro, com boa qualidade de vida ${ }^{9,22,30,31}$.

\section{CONSIDERAÇÕES FINAIS}

No Brasil, o Sistema Único de Saúde (SUS), apesar dos poucos centros de atendimento especializado, da demora de conseguir as cirurgias de redesignação sexual, ainda está mais capacitado para receber, acolher e atender a pessoa transexual. Já o atendimento privado, nesta área, está despreparado, com raríssimas exceções.

O tratamento ambulatorial com hormônios pode ser realizado pela(o) profissional apta(o) a fazê-lo, geralmente clínico, endocrinologista, ginecologista e urologista.

Em casos de enfermidades específicas, deverão ser encaminhados para o especialista. Considerando que a comunidade transexual está aumentando significativamente, há necessidade de um maior número de pesquisas científicas na área da transexualidade e da inclusão deste tema na graduação dos profissionais da saúde. Através destas atitudes é que haverá uma adequada formação dos profissionais, em geral, pois a pessoa transexual necessita de atendimento médico em todas as especialidades, não somente naquelas relacionadas à transexualidade, assim, todos os/as profissionais da saúde têm que estar aptos(as) a prestar um atendimento adequado.

Os sistemas de saúde público e privado têm que constantemente se atualizarem no sentido de como receber, acolher e tratar pacientes transexuais, com suas necessidades individuais.

\section{REFERÊNCIAS}

1. American Medical Association AMA. Resolution: 122 (A-08). 2008.

2. Anton BS. Proceedings of the American Psychological Association for the legislative year 2008: Minutes of the annual meeting of the Council of Representatives, February 22-24, 2008, Washington, DC, and August 13 
and 17, 2008, Boston, MA, and minutes of the February. Am Psychol. 2009;64(5):372-453.

3. Coleman E, Bockting W, Botzer M, Cohen-Kettenis $P$, DeCuypere G, Feldman J, et al. Standards of Care for the Health of Transsexual, Transgender, and GenderNonconforming People, Version 7. Int J Transgenderism. $2012 ; 13(4): 165-232$.

4. Scott, J. Gênero: uma categoria útil para análise histórica. Educação e Realidade. Porto Alegre, 1995; 20(2):71-99.

5. Butler J. Problemas de gênero: feminismo e subversão de identidade. 6a Ed. Civilização Brasileira. Rio de Janeiro, 2013.

6. Bento, B. A reinvenção do corpo: sexualidade e gênero na experiência transexual. Ed. Garamond. Rio de Janeiro, 2006.

7. Arcelus J, Bouman WP, Van Den Noortgate W, Claes L, Witcomb G, Fernandez-Aranda F. Systematic review and meta-analysis of prevalence studies in transsexualism. Eur Psychiatry. 2015;30(6):807-15.

8. Meerwijk EL, Sevelius JM. Transgender population size in the United States: A meta-regression of population-based probability samples. Am J Public Health. 2017;107(2):e1-8.

9. Cohen-Kettenis PT, Klink D. Adolescents with gender dysphoria. Best Pract Res Clin Endocrinol Metab. 2015 Jun;29(3):485-95.

10. Jesus JG de. Orientações Sobre Identidade De Gênero: Conceitos E Termos. 2012. 23 p.

11. Peres WS, Toledo LG. Dissidências existenciais de gênero: resistências e enfrentamentos ao biopoder. Rev Psicol Política. 2011;11(22):261-77.

12. Winter S, Diamond M, Green J, Karasic D, Reed T, Whittle $\mathrm{S}$, et al. Transgender people: health at the margins of society. Lancet. 2016 Jul;388(10042):390-400.

13. World Health Organization. ICD-11.

14. Medicina CF de. RESOLUÇÃO CFM n 2.265/2019. 2020;(D).

15. Sociedade Brasileira de Endocrinologia e Metabologia SBEM. Resolução do CFM e Incongruência de Gênero. 2020.

16. Vance SR, Ehrensaft D, Rosenthal SM. Psychological and Medical Care of Gender Nonconforming Youth. Pediatrics. 2014 Dec;134(6):1184-92.

17. Colizzi M, Costa R, Todarello O. Dissociative symptoms in individuals with gender dysphoria: Is the elevated prevalence real? Psychiatry Res. 2015 Mar;226(1):173-80.

18. Ministério da Saúde. Sistema de Informação de Agravos de Notificação - SISNAN. 2012. p. 23054.
19. Secretaria de Estado da Saúde do Paraná -, SESA. Protocolo de Atendimento Centro de Pesquisa e Atendimento a Travestis e Transexuais. Curitiba: Secretaria de Estado da Saúde do Paraná; 2018. p. 25.

20. Chipkin SR, Kim F. Ten Most Important Things to Know About Caring for Transgender Patients. Vol. 130, American Journal of Medicine. Elsevier; 2017. p. 1238-45.

21. Safer JD, Coleman E, Feldman J, Garofalo R, Hembree W, Radix A, et al. Barriers to healthcare for transgender individuals. Curr Opin Endocrinol Diabetes Obes. 2016 Apr;23(2):168-71.

22. Spizzirri G, Ankier C, Abdo CHN. Considerações sobre 0 atendimento aos indivíduos transgêneros. Diagn Trat. 2017;22(4):176-9.

23. Hembree WC, Cohen-Kettenis PT, Gooren L, Hannema $\mathrm{SE}$, Meyer WJ, Murad $\mathrm{MH}$, et al. Endocrine treatment of gender-dysphoric/ gender-incongruent persons: An endocrine society*clinical practice guideline. J Clin Endocrinol Metab. 2017;102(11):3869-903.

24. Ato presidencial. Atos do Poder Executivo. 2016;

25. Mehta PK, Easter SR, Potter J, Castleberry N, Schulkin J, Robinson JN. Lesbian, Gay, Bisexual, and Transgender Health: Obstetrician-Gynecologists' Training, Attitudes, Knowledge, and Practice. J Women's Heal. 2018 Dec;27(12):1459-65.

26. INSTITUTO NACIONAL DE CÂNCER. Estatísticas de câncer | INCA - Instituto Nacional de Câncer. 01/08/2019. 2019.

27. Deebel NA, Morin JP, Autorino R, Vince R, Grob B, Hampton LJ. Prostate Cancer in Transgender Women: Incidence, Etiopathogenesis, and Management Challenges. Urology. 2017 Dec;110:166-71.

28. Dandona $P$, Rosenberg MT. A practical guide to male hypogonadism in the primary care setting. Int J Clin Pract. 2010;64(6):682-96.

29. Associação Nacional de Travestis e Transexuais ANTRA. Mapa os Assassinatos de Travestis e Transexuais no Brasil. 2017. p. 121.

30. Roberts $A L$, Rosario $M$, Corliss $H L$, Koenen KC, Austin SB. Childhood Gender Nonconformity: A Risk Indicator for Childhood Abuse and Posttraumatic Stress in Youth. Pediatrics. 2012 Mar;129(3):410-7.

31. Whitman CN, Han H. Clinician competencies: Strengths and limitations for work with transgender and gender non-conforming (TGNC) clients. Int J Transgenderism. 2017 Apr; 18(2):154-71. 


\section{Autores e contribuições:}

Kadija Rahal Chrisostomo (KRC): desenvolvimento de protocolo / projeto, coleta ou gerenciamento de dados, redação / edição do manuscrito.

Bernardo Passos Sobreiro (BS): redação / edição do manuscrito.

Eduardo Rahal Chrisostomo (ERC): coleta ou gerenciamento de dados, redação / edição do manuscrito.

Renato Nisihara (RN): Desenvolvimento de protocolo / projeto, coleta ou gerenciamento de dados, redação / edição do manuscrito.

Divulgação. O autor não relata conflitos de interesse neste trabalho.

Esta pesquisa não recebeu nenhuma bolsa específica de agências de fomento nos setores público, comercial ou sem fins lucrativos.

\section{Autor Correspondente:}

Kadija Rahal Chrisostomo

kadijarc@hotmail.com

Editor:

Prof. Dr. Marcelo Riberto

Recebido: $16 / 12 / 2020$

Aprovado: 18/06/2021 